\title{
Reading in Arabic: New Evidence for the Role of Vowel Signs
}

\author{
Raphiq Ibrahim \\ The Edmond J. Safra Brain Research Center for the Study of Learning Disabilities and Department of Learning \\ Disabilities, University of Haifa, Haifa, Israel \\ Email: raphiq@psy.haifa.ac.il
}

Received December $18^{\text {th }}, 2012$; revised January $19^{\text {th }}, 2013$; accepted February $2^{\text {nd }}, 2013$

\begin{abstract}
Copyright (C) 2013 Raphiq Ibrahim. This is an open access article distributed under the Creative Commons Attribution License, which permits unrestricted use, distribution, and reproduction in any medium, provided the original work is properly cited.
\end{abstract}

\begin{abstract}
The aim of this study was to examine the effect of vowelization on reading Arabic orthography. Native children speakers of Arabic were asked to read aloud words (vowelized and unvowelized) and pseudowords. The results showed that unvowelized words were read aloud more quickly and more accurately than the shallow fully vowelized Arabic words. The disadvantage of vowelized words in both speed and accuracy was therefore unexpected, and, furthermore, inconsistent with findings from several other relevant studies. The results suggested that Arab children used a different perceptual and coding strategy when the stimuli differ in their lexical feature (word vs pseudoword) and visual/orthographic feature (vowelized vs unvowelized).
\end{abstract}

Keywords: Arabic; Words; Pseudoword; Orthography; Transparency; Reading

\section{Introduction}

The ability to decode letters into sounds as a predictor of variability in literacy acquisition was studied for great extent in English (Snowling, 2000). The consistency between letters and sounds called transparency of the orthography and it could determine the ease of word decoding (Ziegler \& Goswami, 2005). Indeed, the transparency of the script has been found to be an important factor when learning to read (Katz \& Frost, 1992; Leong \& Joshi, 1997). In that regard, orthographies can be seen as a continuum of varying degree of consistency between sounds and symbols. In regard to Arabic language, the orthographic transparency theory (Saiegh-Haddad \& Geva, 2008) maintains that the relevance to reading of orthographic mechanisms as against phonological processes is dependent on orthographic transparency. Hence, phonological processes would be used more in reading orthographically shallow vowelized Arabic and Hebrew (as Semetic languages) because the mapping of graphemes to phonemes is consistent unlike unvowelized Arabic and Hebrew which is considered a deep orthography. In deep orthographies, such as unvowelized Arabic and Hebrew script, word decoding necessitates the use of large-unit orthographic units (such as morphemes) for the retrieval of word pronunciation (Aro \& Wimmer, 2003; Saiegh-Haddad \& Geva, 2008).

In 1987, Frost, Katz and Bentin directly tested the Orthographic Depth Hypothesis in a three-way comparison between, Hebrew, Serbo-Croatian and English testing a study of naming and lexical decision times for words versus nonwords. Their study adopted the rational that in shallow orthographies the lexicon plays a minor role in naming process compared to its role in the lexical decision process, hence the greatest difference between naming and lexical decision reaction times should be in Serbo-Coratian, which has the shallowest orthography while deep (unvowelized) Hebrew should show the greatest similarity. The results show that naming times were considerably faster than lexical decision times in Serbo-Coratian but, in Hebrew, lexical decision and naming looked quite similar. Thus, the results support the hypothesis that the shallower the orthography, the greater the amount of phonological recoding that is carried out for naming versus lexical decision. In addition, consistent with the idea that non-oral reading involves less exhaustive phonological processing than oral reading; oral reading rates are relatively slow and lag behind silent reading rates (Barker, Torgesen, \& Wangner, 1992; Frost, 1998). Frost's "large units" are called by Ziegler and Goswami (2005) a large grain sizes. In their Psycholinguistic Grain Size theory, Ziegler and Goswami assume that for a successful phonological recoding, children need to find grain sizes between the orthography and phonology of their languages that allow the most straightforward and least ambiguous mapping between the two domains. Therefore, readers of relatively consistent orthographies can use an exclusively small unit strategy (i.e., grapheme-phoneme correspondences) without making many reading errors. However, while reading less orthographically consistent languages, the reader cannot use smaller grain sizes as easily because inconsistency is much higher for smaller grapheme units than for larger units. So the reader tends to learn additional correspondences for larger orthographic units, such as syllables, rimes or whole words (Treiman, Mullemix, BijeljacBabic, \& Richmond-Welty, 1995).

Ziegler and Goswami (2005) concluded that inconsistent orthographies push readers into developing both small and large units recoding strategies in parallel, supplementing graphemephoneme conversion strategies with the recognition of letter 
patterns for rimes and attempts at whole word recognition, which is also termed the flexible-unit-size hypothesis (Brown \& Deavers, 1999). In the study of Goswami, Ziegler, Dalton and Schneider (2001), their English subjects show much stronger influences from whole-word phonology when reading pseudohomophones. They also showed stronger switching costs when words cannot be decoded using one grain size only (Goswami et al., 2003) and evidence for the adaptive use of large unit and small unit strategies in response to task demands (Brown \& Deavers, 1999).

Based on the above consideration, it can be assumed that the reader needs a well-specified phonological representation for accurate word naming (Frost, 1998; Stanovich \& Stanovich, 1999; Wagner, Torgesen, \& Rashotte, 1994). If readers are having difficulty in decoding words, then their short-term memory may become overloaded and their ability to comprehend sentences could be affected (Shankweiler, 1989). However, if oral word reading and comprehension skills are based on different underlying skills and abilities, then the ability of oral word reading may not predict reading comprehension. Share (2008) claimed that phonology has a reduced role in lexical decision tasks or silent reading compared to oral reading. In their study among third graders, Barker, Torgesen and Wanger (1992) found that phoneme deletion and pseudoword repetition correlated more strongly with oral text reading rate than silent text reading rate. Moreover, Corcos and Willows (1993) concluded that oral naming involves more attention to phonological analysis than orthographic units or meaning relative to silent reading.

Traditionally, oral reading accuracy and fluency are assessed by reading aloud a graded list of words (or pseudowords). These lists are typically graded for length and "difficulty", usually started with short and high-frequency words that become progressively longer and less-frequent words (Fuchs et al., 2001; Share, 2008). When reading aloud, correct pronunciation depends on exhaustively specified phonological representations, and does not necessarily involve access to meaning (Coltheart, 1978). However, there is a need here, to clarify the differences between reading vowelized and unvowelized lists of words. Based on the Orthographic Depth Hypothesis (Frost, 2005), the phonological structure of the printed word in a shallow orthography can be easily recovered from print by applying a simple process of phonological computation. In contrast, in deep orthographies like unvowelized Arabic and Hebrew, readers are encouraged to process printed words by making use of larger units or word morphology via visual-orthographic structure. Although measures of accuracy seem to be good predictors of variability in literacy acquisition of a less transparent script, measures of speed may be better predictors of variability in more transparent scripts (e.g., Landerl et al., 1997). In that regard, previous studies of Ibrahim and colleagues that the graphic characters of the Arabic script constitute a specific challenge to Arabic readers and, in particular, to their ability to distinguish between letters. Two studies by Ibrahim et al. (2002) and Eviatar et al. (2004) found Arabic-Israeli participants slower in processing Arabic letters than Hebrew letters, despite Arabic being reported as the participants' first language. These researchers concluded that this difference was due to the visual/graphic complexity of Arabic script. On the other hand, other studies have concluded that decoding of both Arabic and Hebrew demands more visuo-spatial awareness or visual attention than decoding in English (Share \& Levin, 1999; Shatil \& Share, 2003), and Geva \& Siegel (2000) found that Eng-
lish-Hebrew bilingual children made more visual letter recognition errors in Hebrew than in English.

\section{Orthographic Complexity in Arabic}

In Arabic, all verbs and most nouns are written primarily as consonantal roots that are differently affixed and vowelled to form the words of the lexicon (Berman, 1978). Most written materials in both languages do not include vowels. When vowels do appear (in poetry, children's books and liturgical texts), they are signified by diacritical marks above, below or within the body of the word. Inclusion of these marks completely specifies the phonological form of the orthographic string, making it transparent in terms of orthography/phonology relations. The effects of the omission of vowels on skilled reading in Hebrew has been shown in an interesting study by Shimron and Sivan (1994), who showed that adult Hebrew-English bilinguals read text more quickly in English than in unvowelled Hebrew, but not more quickly than Hebrew text that includes the vowel diacritics. Thus, in Hebrew, even though addition of vowels results in a somewhat more complex visual form of the text, it facilitates both the speed and the comprehension of reading.

In Arabic, there are three diacritics signifying short vowels: two are positioned above the letter: fatHa $(\hat{)})=\mathrm{a}$, damme $(\dot{)})=$ $\mathrm{u}$, and one is positioned below: kasra $(\mathrm{l}) \mathrm{i}$. Although these vowels are not letters, their combinations with consonants form CV syllables. In addition, there are double fatHa $(\overline{)})=$ "an"; double damme $(\stackrel{\circ}{)})=$ "on"; and double kasra $(\mathrm{g})=$ "in". These vowel signs also have a syntactic role as they are used to mark indefinite subjects (e.g., subjects that in English would not be preceded by "the"). For example: fatHa or double fatHa on the last letter of a word signifies it as the object of the sentence, while damme or double damme signifies it as the subject of the sentence. In addition to the diacritics for short vowels, there are four other reading signs: the skoon $(\dot{)})$ which signals absence of a vowel, shada () which signals doubling of a consonant, maddah $(\sim)$ which signals doubling of the letter alif and hamzeh (I) which signals the glottal-stop sound.

There are three letters in Arabic $(ي, 9,1)$ which in addition to signifying specific consonants, also specify long vowels. Thus it can be difficult for the reader to determine whether these dual-function letters represent a vowel or a consonant.

Two additional factors add to the complexity in Arabic. The first has to do with the role of dots. The dots positioned around basic symbols are used to distinguish different letters, and the inclusion of diacritical marks to represent short vowels and additional features such as letter doubling are used to determine syntactic function. Abu-Rabia (1997, 1998) investigated the role of vowelization and its influence on the reading accuracy of poor and normal Arabic readers. The results of these studies indicated that vowelization led to significantly increased accuracy in word reading in both poor and skilled/ normal readers, and similar results were obtained with skilled adult readers (Abu-Rabia, 2001). Shimron (1999) found similar results in. Hebrew where the inclusion of vowel signs in written Hebrew facilitated naming speed. In the Arab world, pupils start to read without diacritical marks from grade 6 onwards and, as such, studies focusing on earlier grades rather than on older children and adults may be more informative of the acquisition of literacy skills under vowelized and non-vowelized conditions. Therefore the current work can be considered unique and more sensitive to the specific linguistic nature of written 
Arabic in that it focuses on early grades of Arabic literacy learning amongst Arabic speakers.

The hypothesis tested in this study is that one of the reasons for slowness in reading acquisition in Arabic in normal readers is due in part to the large perceptual load in vowelized stimuli. Specifically, the study explored whether fully vowelized scripts in word and non-word help or hinder reading. It was hypothesized that reading aloud vowelized isolated words in Arabic would be more accurate than reading unvowelized words due to the simple, consistent and fairly complete connections between graphemes phonemes.

The words used in this experiment were divided to two groups: vowelized and non-vowelized words and non-words. Specifically, it was hypothesized that reading aloud vowelized isolated words in Arabic would be more accurate than reading non-vowelized words due to the simple, consistent and fairly complete connections between graphemes phonemes. Secondly, reading vowelized isolated words in Arabic would be slower than reading non-vowelized words because of the incomplete correspondence between graphemes and phonemes and because of the homographic phenomenon. Thus, the participants' performance in this study could be compared with performance in other languages like Hebrew in which naming has been investigated. On the basis of previous studies of with detecting vowelized Arabic words compared with Hebrew words (Abdelhadi, Ibrahim, \& Eviatar, 2011) and naming performance with vowelized and non-vowelized Hebrew words (Bentin \& Frost, 1987; Frost, 1994), we predicted that naming would be faster for words and would be slowest for pseudowords.

In our recent study (Abdulhadi, Ibrahim, \& Eviatar, 2011), we hypothesized that voweled text may result in perceptual overload, making simple detection of letters and vowels more difficult. We asked children in 3rd and 6th grade, who were identified by their teachers as good readers, to detect a vowel in a three-letter stimulus in Hebrew and in Arabic. In both languages, the target was the diacritic for the vowel "a", which is a small horizontal line that appears above the letter in Arabic and below the letter in Hebrew. The stimuli were: words, nonsense trigrams, and nonletter stimuli. The main and important result was that detection of a vowel target was faster in Hebrew (a language the children are learning, but in which they are not fluent) than in Arabic. We believe that this is because visually, the Hebrew stimuli are less complex then the Arabic stimuli.

\section{Method}

\section{Participants}

Seventy five 8 th graders (42 females and 33 males), recruited from two regular schools in north of Israel. None of the participants suffered from neurological, emotional, or learning disorders. These data was obtained by homeroom teachers, school counselors and psychologists. Students diagnosed as suffering from learning disabilities, ADHD, ADD or other neurological disorders, were excluded from the sample. All the children in the study verbally expressed willingness to participate.

\section{Materials}

Reading measures were constructed to examine the ability to read isolated Arabic words and non-words. Three tests provided measures of decoding accuracy and reading time for vowelized words, unvowelized words and pseudowords. Subjects were asked to read as quickly and accurately as possible. Scores for reading rate (or fluency) were based on the number of words or pseudowords read correctly. In order to obtain a composite decoding score, Z-scores were calculated for each of the three tasks (i.e., vowelized, unvowelized words and pseudowords) then averaged.

A list of 50 words was arranged in order of increasing length (3 - 6 syllables) and decreasing frequency for fully vowelized word naming. A list of 50 different words arranged in order of increasing length (3 - 6 syllables) and decreasing frequency for unvowelized word naming. The frequency of the two lists were equalized by language teachers from the same schools. A list of 50 pseudowords was arranged in order of increasing length (3 6 syllables) for pseudoword naming. See examples of stimuli in Table 1.

\section{Procedure}

For the assessment of word reading, participants were tested individually in a quiet room in school. The three isolated word lists were administered in the following order: vowelized words, pseudowords and unvowelized words. Total reading time and accuracy rate were measured for each list.

\section{Results}

The data were initially analyzed with repeated measures analysis used to test differences in reading time and reading accuracy between the three reading tasks.

Repeated measures analyses were conducted to determine whether reading speed and accuracy differed among the three word types (pseudowords, unvowelized and vowelized words). A significant overall effect for "word type" was obtained on measures of speed $(\mathrm{F}(2,15)=305.57, p<0.001)$. Follow-up pair-wise comparisons revealed significant differences between the three types; between pseudowords and fully vowelized word naming speed, $(\mathrm{F}(1,74)=209.02, p<0.001$, and between pseudowords and unvowelized word naming speed, $(\mathrm{F}(1,74)$ $=413.2, p<0.001)$, and between vowelized and unvowelized word naming $(\mathrm{F}(1,74)=191.45, p<0.001)$. These results indicate that the Arabic-speaking students were slowest in reading pseudowords, and fastest in reading unvowelized words, with vowelized word naming speed in between.

Reading accuracy for these same three lists revealed a similar pattern. The overall analysis was again found to be significant, $\mathrm{F}(2,15)=115.83, p<0.001$. Significant differences were found between pseudowords and fully vowelized word naming accuracy, $\mathrm{F}(1,74)=44.13, p<0.001$; pseudowords and unvowelized word naming accuracy, $\mathrm{F}(1,74)=162.8, p<0.001$, and between vowelized and unvowelized word accuracy, $\mathrm{F}(1,74)=$ $134.44, p<0.001$. These results indicate that the Arabicspeaking students made the highest mean number of errors in

Table 1.

Examples of vowelized and unvowelized Arabic words.

\begin{tabular}{|c|c|c|}
\hline Type of stimuli & Unvowelized & Vowelized \\
\hline Three letters & & آرَريءٌ barêun/ \\
\hline Four letters & وفاق/wefak^/ & سبباقُ /sebak^un/ \\
\hline Five letters & /fareda/ فريدة & 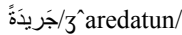 \\
\hline
\end{tabular}


reading pseudowords, the smallest mean number of errors in reading unvowelized words, with, once again, vowelized word errors in between.

\section{Discussion}

The aim of this study was to examine the effect of vowelization on reading Arabic orthography. The relationships between oral word (vowelized and unvowelized) and pseudoword reading were examined. The results showed that among Arabic readers, pseudoword reading was the slowest and least accurate, reading unvowelized words was the fastest and the most accurate, while reading vowelized word naming speed and accuracy in between. In contrary to the finding mentioned above, the finding that pseudowords are named more slowly and less accurately than real words confirm in the "word-superiority" effect by assuming that words may access the lexicon "directly" by using whole-word orthographic codes, thereby permitting direct access to whole-word phonological information (Coltheart, 1978; Bentin \& Ibrahim, 1996). However, when reading pseudowords, the reader must rely extensively on letter-sound conversion rules (Taouk \& Coltheart, 2004, Simon, Bernard, Lalonde \& Rebai, 2004). This process of pre-lexical phonological assembly is slower and less efficient (e.g., Coltheart, Besner, Jonasson, \& Davelaar, 1979; Seidenberg \& Zevin, 2006). Surprisingly, unvowelized words were read aloud more quickly and more accurately than the shallow fully vowelized Arabic words. It was expected that readers would be able to achieve greater pronunciation accuracy through exclusive reliance on the phonological information offered by diacritics that disambiguate Arabic homographs (Azzam, 1989; Abu-Rabia \& Siegel, 1995; Abu-Rabia, 1996, 1998; Abu-Rabia et al., 2003; Frost, 1994, 1995; Saiegh-Haddad, 2003; Saiegh-Haddad \& Geva, 2008), which are often phonologically and semantically ambiguous. Furthermore, in reading unvowelized words the reader must activate his or her knowledge of literary Arabic such as vocabulary, morphology, and exposure to print (AbuRabia, 1996, 1998; Abu-Rabia et al., 2003; Frost, 1994; Shimron, 1999). This is especially true in reading a list of isolated words without short vowels because the reader is deprived of contextual information that is known to facilitate word identification (Perfetti, 1985; Rumelhart, 1977). In fully vowelized Arabic, vowels supply a regular and consistent representation that renders any additional linguistic information redundant, especially when readers are not timed (Saiegh-Haddad \& Geva, 2008). In unvowelized Arabic, however, all diacritical marks are absent and vowel identity has to be restored by the reader as an integral part of the word identification process. The disadvantage of vowelized words in both speed and accuracy was therefore unexpected, and, furthermore, inconsistent with findings from several other relevant studies. For example, AbuRabia reported a series of studies examining reading accuracy as a function of vowels and context (Abu-Rabia \& Siegel, 1995; Abu-Rabia, 1996, 1998). The results consistently indicated that vowels improved the reading accuracy in both poor and skilled readers, in young Arabic speakers (8th grade), as well as for highly proficient adult readers (university students). There are several possible explanations for this mismatch between the present finding and Abu-Rabia's results. First, all of the preceding studies tested only reading accuracy but not reading speed. The tests in Abu-Rabia's studies were not timed, while in the present study, the participants were asked to read as quickly and accurately as they could. This raises the possibility of a speed-accuracy trade-off such that accuracy was sacrificed for speed. However, this account can be readily dismissed because the present results showed a significant positive correlation between reading time and accuracy in each task. Second, as mentioned above, comprehensive phonological information is required for accurate pronunciation. However, the acquisition of this information demands that the reader recognize all the different short-vowel diacritics that are located above, and/or in, and/or below the letters. The participants in this study typically have had no contact with vowelized Arabic script for several years because by the 4th grade vowelized texts are gradually phased out. Consistently, almost all the participants in the present study reported difficulties reading the vowelized Arabic lists employed here, explicitly stating that the vowel signs constituted a hindrance to them. Similarly, when Abu-Rabia and Siegel (2003) tested oral word read ability using vowelized words, their 8th grade participants had the same complaint. In this line, Roman and Pavard (1987) conducted eye movement studies on two Arabic texts, vowelized and unvowelized. Findings showed that the text reading processes seem to be impaired when vowels are introduced. Vowels significantly reduced reading speed and significantly increased the number of fixations as well as fixation duration. In a follow-up experiment, Roman and Pavard (1987) investigated the issue of lexical access in Arabic again using vowelized and unvowelized words among skilled readers. Results showed that the presence of vowels significantly increased lexical decision latency, delaying responses by $300 \mathrm{msec}$. The researchers concluded that the addition of phonologically disambiguating vowel points inhibits rather than facilitates lexical decision, and that readers need to use more contextual information in order to access Arabic words.

Third, word recognition abilities rest on phonological and orthographic skills (Frith, 1985), and as readers become more proficient they rely more on the use of visual orthographic information (i.e., spelling representations or orthographic codes) than phonological decoding processes and word recognition (Fender, 2008). Therefore, skilled 8th grade readers of Arabic may be shifting away from phonological recoding (which is helped by vowelized script) toward an orthographic phase in which unvowelized words that do not contain a complete representation of the individual sounds, can be recognized directly in their visual form, rather than indirectly in terms of their pronunciations (Taouk \& Coltheart, 2004). The subjects in this study were skilled readers, used to reading unvowelized but not vowelized script. Their presumably extensive orthographic knowledge predisposes their reading strategy to be primarily visual-orthographic rather than phonological (Abu-Rabia et al., 2003). Consistent with this hypothesis, Azzam (1993) examined the reading and spelling errors committed by 150 Arabic primary-school children (6 - 11 years old). Azzam's results provided evidence for a transition from phonological-recoding phase, to an orthographic phase mainly reliant on a direct whole-word encoding strategy. In addition, Taouk and Coltheart (2004), set out to determine the degree to which children and adults differentially use phonological recoding and whole-word encoding strategies in reading. They suggest that if skilled adult readers rely on a direct whole-word encoding procedure for reading aloud, and less skilled children readers rely on an indirect recoding procedure that makes use of letter-sound conversion rules, then it would be expected that adults would have 
greater difficulty reading real words consisting of letters written in their incorrect positions (كَلْب اكلْبِ ) than children with adequate knowledge of letter-sound rules. The results indicated that adults were much more disadvantaged than children by the illegal positioning of letters that are foreign to the standard orthographic form of the word.

In addition, since skilled readers of Arabic normally read words without short vowels, they recognize ambiguous words by relying on other resources, such as morphological and contextual knowledge. In the present study, however, the words were presented without sentence context depriving the reader of morpho-syntactic information and forcing the reader to rely on the phonological information supplied in the no-longer-familiar vowels. If we assume that skilled readers favor reading words through visual-orthographic information then the unvowelized forms they typically read will be read faster and more accurately than the vowelized words.

\section{Conclusion}

Reading in the Arabic language is found to be uniquely challenging due to linguistic and visual factors. The results reported above suggest that 8 th graders used a different perceptual and coding strategy when the stimuli differ in their lexical feature (word vs pseudoword) and visual/orthographic feature (vowelized vs unvowelized). The present research adds to the accumulating body of evidence affirming the cognitive complexity of written Arabic.

The main findings of the present study have general implications for teaching reading in Arabic, both for native Arabic speakers and for learners who are not Arabic native speakers. In that regard, Arab schools have the responsibility to consider encouraging reading in every possible way, such as designing a library in every classroom, giving reading homework, holding reading contests, and holding discussions with students on books they have read. Second, it is recommended to increase the hours of teaching Arabic language, especially as the Arab pupils begin to study a second language (Hebrew) in second grade and third language (English) in third grade, often at the expense of the hours allocated to teaching the Arabic language.

\section{REFERENCES}

Abdelhadi, S., Ibrahim, R., \& Eviatar. Z. (2011). Perceptual load in the reading of Arabic: Effects of orthographic visual complexity on detection. Writing Systems Research, 3, 117-127. doi:10.1093/wsr/wsr014

Abu-Rabia, S. (1997). Reading in Arabic orthography: The effects of vowels and context on reading accuracy of poor and skilled Arabic native readers in reading paragraphs, sentences, and isolated words. Journal of Psycholinguistic Research, 26, 465-482. doi:10.1023/A:1025034220924

Abu-Rabia, S. (1996). The role of vowels and context in reading of highly skilled native Arabic readers. Journal of Psycholinguistic Research, 25, 629-641. doi:10.1007/BF01712413

Abu-Rabia, S. (1998). Reading Arabic texts: Effects of text type, reader type and vowelization. Reading and Writing, 10, 105-119. doi:10.1023/A:1007906222227

Abu-Rabia, S. (2001). The role of vowels in reading Semitic scripts: Data from Arabic and Hebrew. Reading and Writing, 14, 39-59. doi:10.1023/A:1008147606320

Abu-Rabia, S., Share, D., \& Mansour, M. S. (2003). Word recognition and basic cognitive processes among reading-disabled and normal readers in Arabic. Reading and Writing, 16, 423-442.
doi:10.1023/A:1024237415143

Abu-Rabia, S., \& Siegel, L. S. (1995). Different orthographies, different context effects: The effects of Arabic sentence context in skilled and poor readers. Reading Psychology: An International Quarterly, 16, 1-19. doi:10.1080/0270271950160101

Aro, M., \& Wimmer, H. (2003). Learning to read: English in comparison to six more regular orthographies. Applied Psycholinguistics, 24, 621-635. doi:10.1017/S0142716403000316

Azzam, R. (1989). In the linguistics of literacy, orthography and reading of the Arabic language. In J. Aaron, \& R. M. Joshi (Eds.), Reading and Writing Disorders in Different Orthographic Systems (pp. 203-218). Berlin: Springer. doi:10.1007/978-94-009-1041-6 12

Azzam, R. (1993). The nature of Arabic reading and spelling errors of young children. Reading and Writing, 5, 355-385. doi:10.1007/BF01043112

Barker, A., Torgesen, K., \& Wagner, K. (1992). The role of orthographic processing skills on five different reading tasks. Reading Research Quarterly, 27, 334-345. doi:10.2307/747673

Bentin, S., \& Ibrahim, R. (1996). New evidence for phonological processing during visual word recognition: The case of Arabic. Journal of Experimental Psychology: Learning, Memory, and Cognition, 22, 309-323. doi:10.1037/0278-7393.22.2.309

Bentin, S., \& Frost, R. (1987). Processing lexical ambiguity and visual word recognition in a deep orthography. Memory \& Cognition, 15, 13-23. doi:10.3758/BF03197708

Berman, R. A. (1978). Modern Hebrew structure. Tel Aviv: University Publisher.

Brown, G. D. A., \& Deavers, R. P. (1999). Units of analysis in nonword reading: Evidence from children and adults. Journal of Experimental Child Psychology, 73, 208-242. doi:10.1006/jecp.1999.2502

Coltheart, M. (1978). Lexical access in simple reading tasks. In: G. Underwood (Ed.), Strategies of Information Processing (pp. 151216). San Diego, CA: Academic Press.

Coltheart, M., Besner, D., Jonasson, J. T., \& Davelaar, E. (1979). Phonological recording in the lexical decision task. Quarterly Journal of experimental Psychology, 31, 489-507. doi: $10.1080 / 14640747908400741$

Corcos, E., \& Willows, D. M. (1993). The processing of orthographic information. In D. M. Willows, R. S. Kruk, \& E. Corcos (Eds.), Visual Processes in Reading and Reading Disabilities (pp. 163-188). Dordrecht/Boston/London: Kluwer Academic Publishers.

Eviatar, Z., Ibrahim, R., \& Ganayim, D. (2004). Orthography and the hemispheres: Visual and linguistic aspects of letter processing. Neuropsychology, 18, 174-184. doi:10.1037/0894-4105.18.1.174

Fender, M. (2008). Spelling knowledge and reading development: Insights from Arab ESL learners. Reading in a Foreign Language, $20,19-42$.

Frith, U. (1985). Beneath the surface of developmental dyslexia. In K. patterson, J. Marshall, \& M. Clotheart (Eds.), Surface Dyslexia (pp. 301-330). Hillsdale, NJ: Erlbaum.

Frost, R. (1994). Prelexical and postlexical strategies in reading: Evidence from deep and shallow orthography. Journal of Experimental Psychology: Learning, Memory, and Cognition, 20, 116-129. doi: $10.1037 / 0278-7393.20 .1 .116$

Frost, R. (1995). Phonological computation and missing vowels: Mapping lexical involvement in reading. Journal of Experimental Psychology: Learning, Memory, and Cognition, 21, 398-408. doi: $10.1037 / 0278-7393.21 .2 .398$

Frost, R. (1998). Toward a strong phonological theory of visual word recognition: True issues and false trails. Psychological Bulletin, 123, 71-99. doi: 10.1037/0033-2909.123.1.71

Frost, R. (2005). Orthographic systems and skilled word recognition processes in reading. In M. J. Snowling, \& C. Hulme (Eds.), The Science of Reading: A Handbook (pp. 272-295). Oxford: Blackwell. doi:10.1002/9780470757642.ch15

Frost, R., Katz, L., \& Bentin, S. (1987). Strategies for visual word recognition and orthogrsphical depth: A multilingual comparison. Journal of Experimental Psychology: Human Perception and Performance, 13, 104-114. doi:10.1037/0096-1523.13.1.104 


\section{R. IBRAHIM}

Fuchs, L. S., Fuchs, D., Hosp, M. K., \& Jenkins, J. R. (2001). Oral reading fluency as an indicator of reading competence: A theoretical, empirical, and historical analysis. Scientific Studies of Reading, 5, 241-258. doi:10.1207/S1532799XSSR0503_3

Geva, E., \& Siegel, L. (2000). Orthographic factors in the concurrent development of basic reading skills in two languages. Reading and Writing, 12, 1-30. doi:10.1023/A:1008017710115

Goswami, U., Ziegler, J. C., Dalton, L., \& Schneider, W. (2001). Children's orthographic representation in English and Greek. European Journal of Psychology of Education, 3, 273-292.

Goswami, U., Ziegler, J. C., Dalton, L., \& Schneider, W. (2003). Nonword reading across orthographies: How flexible is the choice of reading units? Applied Psycholinguistics, 24, 235-247. doi: $10.1017 / \mathrm{S} 0142716403000134$

Goswami, U., \& Ziegler, J. C. (2005). Reading acquisition, development dyslexia, and skilled reading languages: A psycholinguistic grain size theory. Psychological Bulletin, 131, 3-29. doi:10.1037/0033-2909.131.1.3

Ibrahim, R., \& Eviatar, Z., \& Aharon-Peretz, J. (2002). The characteristics of Arabic orthography slows its processing. Neurosychology, 16, 322-326. doi:10.1037/0894-4105.16.3.322

Katz, L., \& Frost, R. (1992). The reading process is different for different orthographies: The orthographic depth hypothesis. In R. Frost, \& L. Katz (Eds.), Orthography, Phonology, Morphology and Meaning (pp. 67-84). Amsterdam: North-Holland.

doi:10.1016/S0166-4115(08)62789-2

Landerl, K., Wimmer, H., \& Frith, U. (1997). The impact of orthographic consistency on dyslexia: A German-English comparison. Cognition, 63, 315-334. doi:10.1016/S0010-0277(97)00005-X

Leong, C. K., \& Joshi, R. M. (1997). Cross-language studies of learning to read and spell: Phonologic and orthographic processing. Dordrecht: Kluwer Academic Press. doi:10.1007/978-94-017-1197-5

Perfetti, C. A. (1985). Reading ability. Oxford: Oxford University Press.

Roman, G., \& Pavard, B. (1987). A comparative study: How we read Arabic and French. In J. K. O’Regan, \& A. Levy-Schoen (Eds.), Eye movements: From physiology to cognition (pp. 431-440). Amsterdam: North Holland Elsevier.

Saiegh-Haddad, E. (2003). Linguistic distance and initial reading acquisition: The case of Arabic diglossia. Applied Psycholinguistics, 24, 431-451. doi:10.1017/S0142716403000225

Saiegh-Haddad, E., \& Geva, E. (2008). Morphological awareness, phonological awareness, and reading in English-Arabic bilingual children. Reading and Writing, 21, 481-504. doi:10.1007/s11145-007-9074-X

Shankweiler, D. (1989). How problems of comprehension are related to difficulties in word reading. In D. Shankweiler, \& I. Y. Liberman (Eds.), Phonology and Reading Disability: Solving the Reading Puzzle (pp. 35-68). Ann Arbor, MI: University of Michigan Press.

Share, D. L., \& Levin, I. (1999). Learning to read and write in Hebrew. In M. Harris, \& G. Hatano (Eds.), Learning to Read and Write: A Cross-Linguistic Perspective (pp. 98-111). New York: Cambridge University Press.

Shatil, E., \& Share, D. (2003). Cognitive antecedents of early reading ability: A test of the modularity hypothesis. Journal of Experimental Child Psychology, 86, 1-31. doi:10.1016/S0022-0965(03)00106-1

Share, D. L. (2008). On the Anglocentricities of Current Reading Research and Practice: The Perils of Overreliance on an "outlier" orthography. Psychological Bulletin, 134, 584-615. doi:10.1037/0033-2909.134.4.584

Shimron, J. (1999). The role of vowel signs in Hebrew: Beyond word recognition. Reading and Writing: An Interdisciplinary Journal, 11, 301-319. doi:10.1023/A:1008045316692

Shimron, J., \& Sivan, T. (1994). Reading proficiency and orthography: Evidence from Hebrew and English. Language Learning, 44, 5-27.

Snowling, M. J. (2000). Dyslexia, second edition. Oxford: Blackwell.

Seidenberg, M. S., \& Zevin, J. D. (2006). Connectionist models in developmental cognitive neuroscience: Critical periods and the paradox of success. In Y. Munakata, \& M. Johnson (Eds.), Attention \& Performance XXI: Processes of Change in Brain and Cognitive Development (pp. 585-612). Oxford: Oxford University Press.

Stanovich, K. E., \& Stanovich, P. (1999). How research might inform the debate about early reading acquisition. In J. V. Oakhill, \& R. Beard (Eds.), Reading Development and the Teaching of Reading (13-31). Oxford: Basil Blackwell.

Taouk, M., \& Coltheart, M. (2004). The cognitive processes involved in learning to read in Arabic. Reading and Writing: An Interdisciplinary Journal, 17, 27-57. doi:10.1023/B:READ.0000013831.91795.ec

Treiman, R., Mullennix, J., Bijeljac-Babic, R., \& Richmond-Welty, E. D. (1995). The special role of rimes in the description, use, and acquisition of English orthography. Journal of Experimental Psychology General, 124, 107-136. doi:10.1037/0096-3445.124.2.107

Wagner, R. K., Torgesen, J. K., \& Rashotte, C. A. (1994). Development of reading-related phonological processing abilities: New evidence of bi-directional causality from a latent variable longitudinal study. Development Psychology, 30, 73-87. doi:10.1037/0012-1649.30.1.73

Ziegler, J. C., \& Goswami, U. (2005). Reading acquisition, developmental dyslexia, and skilled reading across languages: A psycholinguistic grain size theory. Psychological Bulletin, 131, 3-29. doi:10.1037/0033-2909.131.1.3 